ISSN: 1130-3743 - e-ISSN: 2386-5660

DOI: http://dx.doi.org/10.14201/teoredu292167184

\title{
¿LA EDUCACIÓN CAMBIA? REPENSANDO EL SENTIDO Y FINALIDAD DE UNA ESCUELA PARA TODOS
}

\author{
Is education changing? A reflection on the function \\ and meaning of school for everyone
}

\section{Est-il en train de changer l'éducation? Une réflexion sur la fonction et le sens de l'école pour tous}

Roberto SAnz PONCE y Ángela SERRANO SARMIENTO

Universidad Católica de Valencia. Facultad de Magisterio y Ciencias

de la Educación. C/ Sagrado Corazón, 5. 46110 Godella (Valencia).

roberto.sanz@ucv.es; angela.serrano@ucv.es

Fecha de recepción: enero de 2017

Fecha de aceptación: marzo de 2017

RESUMEN

Este artículo reflexiona sobre la función y sentido de la escuela actual. Analiza algunos enfoques aportados por instituciones y pedagogos importantes -UNESCO, Nussbaum, Perrenoud, Robinsson, Morin y Gardner-, abordando temas como la actual crisis educativa o cómo educar hoy. Desde el enfoque de capacidades enfatiza la necesidad de educar a la persona desde su contexto, dando un enfoque al currículo inclusivo. Además, plantea la importancia de una educación humanizadora, centrada en la realidad del alumnado, con un modelo que responda a lo que es capaz de desarrollar la escuela. Una educación basada en un modelo que da importancia a formar y no solo a instruir. El artículo presenta conclusiones sobre la práctica educativa que giran sobre: el rol docente, la relación maestro-alumno, el clima de aula, la atención a la diversidad o las metodologías; temas trasversales a tratar cuando se necesita dar un nuevo sentido al acto de educar. 
Palabras clave: fines de la educación; desarrollo de capacidades; implicaciones pedagógicas; retos de la educación; docentes.

\section{SUMMARY}

In this article, the authors make a reflection on the function and meaning of current school. They analyze the approaches from the significant institutions and pedagogues -UNESCO, Nussbaum, Perrenoud, Robinsson, Morin and Gardner-focusing on the current educational crisis or how educating today. From a capabilities perspective, the authors, they emphasized on the need to educate the person from reality and its context, giving a new approach to educational curriculum from a more inclusive approach. Thus the authors suggest the importance of a humanist education focused on the reality of students, with an educational model that responds to the reality of what school is able to do. An education based on a model that gives importance to formation and not only to instruct. This article also presents conclusions about educational practice as the teacher's role, the teacher-student relationship, the classroom atmosphere, attention to diversity or methodologies; transversal topics when you need to give new meaning to the act of educating.

Key words: education goals; capabilities development; pedagogical consequences; educational challenges; teachers.

\section{SOMMAIRE}

Dans cet article, les auteurs font une réflexion sur la fonction et le sens de l'école actuelle. Ils analysent les approches des institutions et de quelques-uns des pédagogues les plus significatifs -UNESCO, Nussbaum, Perrenoud, Robinsson, Morin et Gardner- et abordent des questions telles quelle la crise éducative ou l'éducation actuelle. Du point de vue des capacités, les auteurs se concentrent sur la nécessité d'éduquer la personne à partir de la réalité et de son contexte, en donnant une nouvelle approche au curriculum éducatif à partir d'une approche plus inclusive. Ainsi, les auteurs suggèrent l'importance d'une éducation humaniste centrée sur la réalité des élèves, avec un modèle éducatif qui réponde à la réalité de ce que l'école est capable de faire. Une éducation basée sur un modèle qui donne de l'importance à la formation et pas seulement à l'instruction. Cet article présente également des conclusions sur la pratique éducative, comme le rôle de l'enseignant, la relation enseignant-élève, l'atmosphère en classe, l'attention à la diversité ou encore les méthodologies transversales lorsque vous avez besoin de donner un nouveau sens à l'acte d'éducation.

Mots clés: objectifs de l'éducation; développement des capacités; implications pédagogiques; défis de l'éducation; enseignants. 


\section{INTRODUCCIÓN}

El desarrollo de los pueblos tiene relación directa con el desarrollo de las personas que los conforman. Esta relación hace referencia -o debería hacerla- al desarrollo de "todas las personas", sin distinciones. Y, es más, esta premisa debería aplicarse con especial atención en aquellos colectivos que se encuentran en situación de vulnerabilidad -económica, cultural, laboral, etc.- o en riesgo de exclusión social.

Los últimos Informes internacionales sobre Desarrollo Humano (PNUD, 2004, 2005, 2009 y 2010) manifiestan que «la verdadera riqueza de una nación está en su gente», por lo que "el objetivo básico del desarrollo es aumentar las libertades humanas en un proceso que puede expandir las capacidades personales, toda vez que amplía las alternativas disponibles para que la gente viva una vida plena y creativa» (PNUD, 2004, 127). Anteriormente, el desarrollo se medía, únicamente, en función de la renta per cápita, presuponiéndose que el aumento del nivel de vida -económica- de un país conllevaba necesariamente al aumento en la calidad de vida -bienestar humano- de todos sus ciudadanos. Esta visión se ha demostrado errónea. El aumento económico puede limitarse a pequeños estratos de la sociedad, manteniéndose grandes bolsas de pobreza o de personas que no ven cubiertas sus necesidades más básicas, a pesar del aumento del Producto Interior Bruto. Según Nussbaum $(2013,13)$, «ese enfoque indiferenciado los alentaba [a los gobiernos] a centrar todos sus esfuerzos en el capítulo del crecimiento económico, sin prestar atención al nivel de vida de sus habitantes más pobres y sin abordar tampoco cuestiones como la salud y la educación, que normalmente no mejoran con el crecimiento sin más». De igual manera, en el devenir histórico el concepto de nivel de vida básico ha cambiado, bien sea considerando vitales bienes superfluos, véase el uso de "móviles»..., o bien sea incluyendo como necesidades básicas un plus al que sólo una parte de la población tiene acceso; por ejemplo: gozar de un Estado de bienestar o exigir una educación determinada, de carácter privado, que convierte la educación de calidad en un bien exclusivo y no global (Sanz y Serrano, 2016).

En esa línea, en el Informe del 2004 (PNUD) se establecían las cuatro capacidades básicas a desarrollar en todas las personas para garantizar una sociedad moderna, democrática y humana: a) vivir una vida larga y saludable; b) tener acceso a la educación; c) tener acceso a los recursos necesarios para disfrutar de un nivel de vida digno; y d) participar en la vida en la comunidad. Tres de estas capacidades hacen referencia a la necesidad de garantizar el derecho a una "vida buena» (Rodríguez, 2006), que responda a la dignidad humana y que permita las relaciones para edificar un futuro común. Pero, en este artículo, nos centraremos fundamentalmente en la segunda de ellas -tener acceso a la educación-, ya que esta articula, posibilita y desarrolla las otras tres. Es, por ello, que entendemos la Educación como una capacidad fértil (Bernal, 2014), que genera la posibilidad de desarrollarse de manera completa. 
En la actualidad el derecho de acceso a la educación solo es reconocido en un porcentaje medio de países, existiendo limitaciones por cuestiones económicas, sociales, étnicas o físicas. La educación debe dotar a todo el alumnado de las capacidades para desarrollar una vida digna y plena, teniendo presentes las posibles necesidades educativas especiales temporales y/o permanentes que cada uno desarrolla en momentos concretos de su vida. Esta intención de poner al servicio de las políticas educativas los recursos y medidas suficientes se encuentra en las agendas de la mayoría de los países desarrollados o en vías de desarrollo, aunque otro asunto es la realidad de sus centros educativos y de su implementación. Por ello, el objetivo de la escuela y de la educación se centra necesariamente en su desarrollo inclusivo y extensivo en una sociedad democrática, libre y plural, que atienda a la realidad funcional de las destrezas y capacidades de cada persona de forma específica y singular.

Bajo estas premisas, hemos analizado algunas propuestas acerca de las capacidades que deben desarrollarse a través de la educación y que suponen un cambio en la concepción de las escuelas y de sus docentes. Capacidades que preparan a las personas para la vida -sin exclusiones-, una vida sostenible y en común en la gran aldea global. Al hilo de estas reflexiones, también hemos introducido algunas de las implicaciones pedagógicas que este cambio conlleva. En definitiva, la pregunta a la que debe responder la Educación ya no es solo en qué educar, sino para qué y cómo hacerlo significativo para el alumnado.

\section{HACIA UNA «NUEVA» NOCIÓN DE ESCUELA}

La escuela es el medio por el cual el Estado garantiza el derecho a la educación. Este derecho, internacionalmente reconocido, tiene su base filosófica en la dignidad humana. La Declaración Universal de los Derechos Humanos (1948) reconoce el derecho que toda persona tiene a desarrollar sus capacidades, por lo que este no puede restringirse, únicamente, a la escolarización, sino que debe garantizar el aprendizaje, es decir, el desarrollo de las capacidades. Esto obliga a las escuelas a perseguir una educación de calidad, mediante una metodología activa, que fomente la curiosidad, la libertad de pensamiento, la creatividad y el pensamiento crítico. Además, debe asentar los valores de cooperación, trabajo en equipo, respeto, autonomía personal y convivencia. Y todo ello atendiendo a todos los alumnos en su diversidad.

Se trata, como se ha dicho, de una "capacidad fértil» (Bernal, 2014, 131), que posibilita la exigencia de políticas públicas y demandas éticas a la altura de la dignidad de la persona (Escámez, 2004). Es, por tanto, un derecho inalienable con una vertiente individual (el desarrollo de la personalidad humana) y otra de carácter social (la convivencia y la cohesión social), por lo que su reconocimiento es un asunto de interés público y político. En esta escuela no es posible el fracaso escolar, ya que debe permitir extraer lo bueno que hay en cada uno, sus talentos y sus tesoros escondidos (Delors, 1996), respetando los ritmos singulares de desarrollo 
individual y de aprendizaje. Entonces: ¿Qué ocurre con aquellos alumnos que aun asistiendo a la escuela no aprenden? ¿Con los que no adquieren las competencias básicas? ¿Con los que expulsamos del sistema? ¿Con los que por sus capacidades diferentes no les permitimos un desarrollo pleno? ¿Estaremos vulnerando su derecho a la educación? ¿Estaremos condenándolos a vivir en una situación de vulnerabilidad o de exclusión social? ¿Estaremos potenciando escuelas no inclusivas?

Una educación del y para el siglo XXI debe responder a este desafío: reconocer el derecho a la educación de todos y ofrecer las mismas oportunidades para desarrollar sus talentos. Y, para ello, debemos entender el derecho a la educación como el derecho a aprender. De esta manera, deberíamos plantearnos una enseñanza "no igualitaria", que atienda a los alumnos como seres únicos, tratando la diversidad dentro de la normalidad. Ken Robinson (2015, 21), en este sentido, afirma que: «Si gestionamos un sistema educativo basado en la normalización y el amoldamiento que anulan la individualidad, la imaginación y la creatividad", no debemos sorprendernos que tanto la individualidad como la imaginación y la creatividad desaparezcan.

No cabe duda de que nuestros alumnos llegan al aula con capacidades y circunstancias vitales que condicionan su manera de afrontar el aprendizaje. ¿Qué hace la escuela? ¿Cómo atiende esta diversidad? ¿Cómo se enfrenta a los diferentes ritmos y maneras de aprender? A estas preguntas debemos responder adaptando los estilos docentes a los estilos de aprendizaje, introduciendo metodologías activas, colaborativas y experienciales, planteándonos la relevancia, pertinencia y equidad del currículum, analizando si da respuesta al reto de preparar para futuros inciertos. En definitiva, transformar la escuela en una institución que siente y aprende, sin olvidar, como afirma Marina (2015), que la tarea docente no es «enseñar», sino conseguir que los alumnos aprendan.

\section{1. ¿Para qué enseñamos? El sentido y la finalidad de la escuela}

¿La escuela prepara para la escuela o prepara para la vida? Si lo analizamos, tal vez nos demos cuenta de que muchos aprendizajes escolares están orientados, únicamente, a la superación de pruebas, pero que tienen escasa transcendencia en la vida diaria y profesional de los alumnos a lo largo de su trayectoria vital (Meirieu, 2003). O puede que no los relacionemos con la vida de tal manera que el contenido carezca de continente, de significancia y, por tanto, de utilidad.

Es por ello que algunas instituciones e investigadores, desde diferentes ámbitos, se preguntan acerca del sentido y pertinencia de los saberes escolares para una sociedad del siglo XXI, así como las capacidades que se deben implementar para construir una sociedad crítica, una ciudadanía más humana e inclusiva, capaz de afrontar los nuevos retos. Pero esta reflexión no está exenta de riesgos y dificultades. Acabar con el «cierre estratégico» (Santos Guerra, 2012), que provoca la excesiva centralización, el irresponsable intervencionismo político en decisiones pedagógicas y curriculares (Gimeno Sacristán, 2000) y el miedo de las escuelas a 
convertirse en "palancas de cambio» no es tarea sencilla si no se tiene claro cuál es la finalidad de la educación.

\subsubsection{El papel de la UnESCO como faro de la educación}

Son muchos los documentos de la UnESCO que abordan temas de calado educativo. Pero son tres los que más transcendencia tienen para plantearnos la finalidad de la educación y de la escuela.

En 1973, Faure y su equipo publicaban Aprender a ser. La educación del futuro, y se planteaban cómo debía ser la educación para afrontar los nuevos desafíos de la sociedad. Mantenían que era preciso "repensar la educación" y que era "necesario que el hombre nuevo esté [estuviese] en condiciones de establecer un equilibrio entre sus capacidades ampliadas de comprensión y de poder, y su contrapartida potencial de orden de carácter afectivo y moral» (Faure, 1973, 45). Esta declaración de intenciones ya evidenciaba un nuevo sentido para la escuela, alejado del establecido en los viejos currícula academicistas, y exigía nuevos aprendizajes: «Aprender a vivir; aprender a aprender, de forma que se puedan ir adquiriendo nuevos conocimientos a lo largo de toda una vida; aprender a pensar de forma libre y crítica; aprender a amar el mundo y a hacerlo más humano; aprender a realizarse en y mediante el trabajo creador» (Faure, 1973, 132).

Años más tarde, Delors y sus colaboradores redactaban el segundo gran informe: La educación encierra un tesoro (1996), donde nuevamente planteaban la finalidad de la educación y de la escuela. El punto de partida era cómo acoplar al ciudadano en la gran aldea planetaria y hacerlo vivir y convivir con sus congéneres, reconociendo sus derechos individuales y colectivos. Y, para ello, establecía los 4 pilares fundamentales -Capacidades- a desarrollar por todos: Aprender a Conocer; Aprender a Hacer; Aprender a vivir juntos/a vivir con los demás; Aprender a Ser. Este informe tenía una clara intencionalidad: "Llevar a cada persona a descubrir, despertar e incrementar sus posibilidades creativas, actualizando así el tesoro escondido en cada uno de nosotros, lo cual supone transcender una visión puramente instrumental de la educación [...], para considerar su función en toda su plenitud, a saber, la realización de la persona que, toda ella, aprende a ser» (Delors, 1996, 96).

El último gran informe, Replantear la educación. ¿Hacia un bien común mundial? (Bokova, 2015), marca como objetivo adaptar la finalidad de la educación al contexto actual, con un espíritu claro: «Es una llamada al diálogo inspirada por una concepción humanista de la educación y el desarrollo que se basa en los principios de respeto a la vida y a la dignidad humana, igualdad de derechos y justicia social, respeto de la diversidad cultural, así como solidaridad internacional y responsabilidad compartida" (Bokova, 2015, 14).

Entre las capacidades a desarrollar por la educación, el informe se aleja de la visión tradicional para establecer la necesidad de: 1) Potenciar la dignidad, capacidad y bienestar personal; 2) Aumentar y mejorar las relaciones con los demás; 3) 
Cuidar la naturaleza y luchar contra el cambio climático; y 4) Desarrollar una serie de competencias blandas y/o transferibles: comunicación, alfabetización digital, resolución de problemas, trabajo en equipo y espíritu de empresa.

\subsection{Algunas propuestas educativas para cambiar la escuela}

\subsubsection{Perrenoud y su escuela para la vida}

Perrenoud (2012) sitúa el debate en los contenidos curriculares que se imparten en la escuela y su utilidad, criticando las políticas educativas actuales que no se cuestionan los saberes escolares y simplemente barnizan levemente cómo enseñarlos.

Por ello, afirma que la escuela actual prepara para continuar con futuros estudios -carácter propedéutico- y no suficientemente para la vida. «Aquellos a los que la escuela debería preparar mejor para la vida son los que saldrán del sistema educativo sin haber adquirido el nivel de cultura suficiente como para aprender fácilmente en la edad adulta lo que no habrán aprendido en la escuela obligatoria" (Perrenoud, 2012, 29). Además, afirma que la institución escolar sitúa en una posición de inferioridad - de vulnerabilidad-a los más necesitados, no cumpliendo con su acometido de niveladora de desigualdades.

Actualmente -continúa diciendo-, existe cierto desfase entre lo que se aprende en la escuela y lo que se necesita en el día a día; hay sobrecarga de contenidos y asignaturas; y olvida que para hacer competentes a los alumnos se necesita de tiempo para la asimilación, puesta en práctica y reflexión de lo aprendido. Por ello, mantiene que «uno no va a la escuela por ir a la escuela, sino para salir de ella provisto de conocimientos, competencias, actitudes, valores que permitan enfrentar la existencia humana" (Perrenoud, 2012, 175). Y entre esos conocimientos, competencias, actitudes, etc., destacan unas competencias transversales y familias de saberes que todos deberían aprender en la escuela obligatoria. Entre dichas competencias sobresalen: saber comunicar, observar, analizar, buscar y clasificar información, adaptarse, innovar, decidir, negociar y argumentar. En cuanto al conjunto de saberes o de situaciones de la vida que conforman el nuevo currículum y dotan al alumnado de aprendizajes para la vida, independientemente de su nivel socioeconómico-cultural o de aquello a lo que se quieran dedicar en el futuro, destacan:

1) Saber defenderse contra las dependencias de cualquier tipo: sustancias, tecnológicas, personales, de los medios de comunicación.

2) Saber construir y hacer evolucionar relaciones de todo tipo con personas de la misma y de las diferentes culturas, actuando de manera civilizada.

3) Saber preservar su "capital salud", a través de una buena y adecuada alimentación, de un modo de vida saludable.

4) Saber orientarse en el mundo laboral, hacia trabajos que les llenen y para los cuales tengan capacidades. 
5) Saber defender sus derechos, frente a la explotación, las injusticias, el acoso o la discriminación.

6) Saber encontrar su lugar, haciendo escuchar sus opiniones, ideas y/o sentimientos, negociando acuerdos y asumiendo responsabilidades.

7) Saber identificar las leyes y los valores, que permitan encontrar un equilibrio entre el bien común y el interés personal.

8) Saber preservar su autonomía, reconocida y preservada frente a cualquier tipo de marginación.

9) Saber aprender rápido cómo comportarse frente a las administraciones.

10) Saber anticipar, construir proyectos y estrategias de futuro y trazar el camino para su consecución.

11) Saber tomar una postura personal ante los problemas del mundo: sostenibilidad, economía, medio ambiente, etc.

12) Saber construir un sentido personal de identidad, pertenencia y valores personales, sin necesidad de recurrir al desprecio y la exclusión.

\subsubsection{Robinson y sus escuelas creativas}

Robinson critica la escuela que potencia la "normalización", anulando así la individualidad y el desarrollo de los talentos personales de cada alumno. Por ello, reclama volver a la enseñanza de lo "esencial», ya que, a su modo de ver, "la finalidad de la educación es capacitar a los alumnos para que comprendan el mundo que les rodea y conozcan sus talentos naturales con el objeto de que puedan realizarse como individuos y convertirse en ciudadanos activos y compasivos» (Robinson, 2015, 24).

Mantiene que los currículos - plagados de asignaturas- solo se centran en un tipo de inteligencia concreta, la inteligencia académica -intelectual-, dejando al margen otras de carácter más creativo. Además, a aquellos alumnos que no destacan en este tipo determinado de inteligencia se les considera "menos capaces", "discapacitados», creyendo que el problema se encuentra, siempre y únicamente, en los propios alumnos y nunca en el sistema. Es por ello que propone una profunda modificación de los contenidos que deben enseñarse y aprenderse en todas las escuelas. Estas nuevas capacidades deberían facilitar a todos los alumnos el tener éxito en la vida y no solo en la propia escuela. Así quedan definidas:

1) Curiosidad. La capacidad de hacer preguntas y de explorar cómo funciona el mundo.

2) Creatividad. La capacidad de generar nuevas ideas y ponerlas en práctica.

3) Crítica. La capacidad de analizar información e ideas, así como de elaborar argumentos y juicios razonados.

4) Comunicación. La capacidad de expresar pensamientos y sentimientos con claridad y confianza en una diversidad de medios y formas. 
5) Colaboración. La capacidad de colaborar constructivamente con otras personas.

6) Compasión. La capacidad de ponerse en el lugar de otras personas y actuar en consecuencia.

7) Calma. La capacidad de conectar con la vida emocional interior y desarrollar un sentido de armonía y equilibrio personal.

8) Civismo. La capacidad de implicarse constructivamente en la sociedad y participar en los procesos que la sustentan.

\subsubsection{Morin y sus siete saberes para la vida}

En 1999, la UNESCO solicita a un grupo de trabajo dirigido por Morin un estudio acerca de aquellos aprendizajes necesarios para la educación del futuro. El informe anuncia la necesidad de una reflexión profunda acerca del sentido y finalidad de la educación en este nuevo milenio. Un mundo incierto e imprevisible precisa, a su juicio, de una educación que ayude a las personas a adaptarse y «derribar las barreras tradicionales entre las disciplinas y concebir la manera de volver a unir lo que hasta ahora ha estado separado" (Morin, 2000, s. p.).

Por tanto, ruptura con las disciplinas tradicionales y apuesta por un aprendizaje globalizado, ya que "nuestra educación nos ha enseñado a separar, compartimentar, aislar y no a ligar los conocimientos, el conjunto de estos constituye un rompecabezas ininteligible» (Morin, 2000, 19), que provoca una «inteligencia miope». Ante esta realidad, destacan 7 saberes necesarios para hacer frente a los desafíos del futuro, favorecer la comprensión del mundo, complejo e incierto, y la resolución de los problemas de manera global.

1) La ceguera del conocimiento: el error y la ilusión. Una de las funciones de la educación sería identificar los errores, las ilusiones y las cegueras derivadas de visiones distorsionadas de la realidad. La escuela debe enseñar a los jóvenes a saber discernir de manera crítica y autónoma lo que sucede en el mundo. "Que ni los hombres ni las mujeres siguieran siendo juguetes inconscientes de sus ideas y de sus propias mentiras. Es un deber de la educación armar a cada uno en el combate vital para la lucidez» (Morin, 2000, 13).

2) Los principios de un conocimiento pertinente. El segundo saber necesario es el conocimiento de los problemas del mundo -políticos, económicos, antropológicos, ecológicos, etc.- para contextualizar correctamente la realidad. La complejidad actual precisa de una actuación decidida de la educación, ya que "hay una inadecuación cada vez más amplia, profunda y grave, por un lado, entre nuestros saberes desunidos, divididos, compartimentados y, por el otro, realidades o problemas cada vez más polidisciplinares, transversales, multidimensionales, transnacionales, globales y planetarios" (Morin, 2000, 15). 
3) Enseñar la condición humana. Otro de los saberes es la necesidad de aprender aquello que es común a la Humanidad, y al mismo tiempo aquello que nos diferencia: la diversidad cultural inherente al ser humano. En este sentido, reclama "para la educación del futuro, de una religación de los conocimientos resultantes de las ciencias naturales con el fin de ubicar la condición humana en el mundo, de los resultantes de las ciencias humanas para aclarar las multi-dimensionalidades y complejidades humanas y la necesidad de integrar el aporte inestimable de las humanidades, no solamente de la filosofía y la historia, sino también de la literatura, la poesía, las artes, etc.» (Morin, 2000, 23).

4) Enseñar la identidad terrenal. El cuarto saber es el aprendizaje de la conciencia y la sensibilidad hacia la Tierra. Por un lado, con la esperanza de crear una "ciudadanía terrestre», capaz de reconocer a los otros y atender sus problemas (pobreza, hambre...). Por otro, hacer consciente de los diferentes peligros que acechan a la Humanidad (armas nucleares, enfermedades contagiosas, drogas...). Y, por último, concienciar sobre la necesidad de cuidar el medioambiente, "civilizar la tierra como casa y jardín de la humanidad" (Morin, 2000, 39). En síntesis, formar una conciencia antropológica; ecológica; cívico-terrenal; y espiritual.

5) Enfrentar las incertidumbres. En un mundo incierto, la escuela debe preparar para saber afrontar con garantías la incertidumbre, adaptarse a esta forma de vida, aprender a convivir con ella y contemplarla como una oportunidad de crecimiento y de desarrollo personal.

6) Enseñar la comprensión. Según Morin, el mundo se enfrenta a una situación paradójica. Por un lado, se encuentra más interrelacionado que nunca y, por otro, existe más incomprensión entre las personas. Hay mucha comunicación y poca empatía. El egocentrismo, el etnocentrismo y el sociocentrismo, base de la xenofobia y el racismo, son las grandes amenazas. «Educar para comprender las matemáticas o cualquier disciplina es una cosa, educar para la comprensión humana es otra; ahí se encuentra justamente la misión espiritual de la educación: enseñar la comprensión entre las personas como condición y garantía de la solidaridad intelectual y moral de la humanidad» (Morin, 2000, 51).

7) La ética del género humano. El último saber es el aprendizaje de una ciudadanía libre y responsable, que dialogue con sus conciudadanos, que se solidariza y que establece y aprende la Democracia como forma de vida. Educar en democracia combate la esclavitud y el totalitarismo, el mercado sin escrúpulos, la ciencia sin ética y la burocracia mal entendida. "La regeneración democrática supone la regeneración del civismo, la regeneración del civismo supone la regeneración de la solidaridad y de la responsabilidad, es decir, el desarrollo de la antropo-ética» (Morin, 2000, 63). 
Todas estas propuestas educativas que engloban el "qué educar» encuentran actualmente su implementación en propuestas metodológicas interesantes, como las que analizamos a continuación.

\subsubsection{Las inteligencias múltiples y las cinco mentes del futuro de Gardner}

Gardner, en sus diferentes obras, hace una crítica a la escuela tradicional, a la que define como "uniforme» $\mathrm{y}$ "unidimensional», basada en una visión limitada y restringida de la inteligencia y con un modelo educativo «en serie» que aporta un mismo currículum para todos, a pesar de las diferencias. Frente a esta visión unitaria de la inteligencia propone una de tipo "polifacético", que dé origen a una escuela diferente que desarrolle capacidades que son importantes para la vida. Una escuela centrada en el individuo, en la persona y no en las áreas curriculares. En ese sentido, "el objetivo de la escuela debería ser el de desarrollar las inteligencias y ayudar a la gente a alcanzar los fines vocacionales y aficiones que se adecuen a su particular espectro de inteligencias» (Gardner, 2015a, 30). Para ello define 7 inteligencias y 5 tipos de mentes que deben ser trabajadas y desarrolladas. Las inteligencias múltiples propuestas por Gardner (2015a) son: 1) Inteligencia lingüística, 2) Lógico-matemática, 3) Espacial, 4) Musical, 5) Corporal-cinética, 6) Interpersonal, 7) Intrapersonal.

De igual manera, sostiene que estos diversos tipos de inteligencias se relacionan con 5 tipos de mentes (Gardner, 2015b) que son: 1) la mente disciplinada, por la que se adquieren los conocimientos, hábitos mentales y pautas de conducta disciplinares y se memorizan los aprendizajes; 2) la mente sintética, que ayuda a sintetizar la información que recibimos; 3) la mente creativa, que permite aportar diferentes formas de actuar y pensar frente a las situaciones a las que nos enfrentamos; 4) la mente respetuosa, que promueve la convivencia con otras personas, aceptando la diversidad y valorándola, y, finalmente, 5) la mente ética, que permite discernir entre lo correcto y lo incorrecto.

\subsubsection{Nussbaum y su propuesta para el desarrollo humano}

Nussbaum presenta una propuesta para el desarrollo humano, en la que responde al principio de dignidad humana. Su enfoque de capacidades se fundamenta sobre tres pilares: a) La calidad de vida de las personas, teniendo como premisa que el trato igualitario no significa necesariamente que se igualen sus condiciones de vida; b) la justicia social, que no coincide siempre con el bien común y tiene sus raíces en la dignidad humana y en las capacidades; y c) ¿qué es capaz de hacer y de ser cada persona? y ¿cuáles son las oportunidades reales para actuar y elegir?

Y, para ello, la educación tiene un papel fundamental.

Nada es más importante para la democracia, para el disfrute de la vida, para la igualdad y la movilidad dentro de la propia nación, para una acción política eficaz 
más allá de las fronteras nacionales. La educación debería concebirse no sólo como una mera aportación de útiles habilidades técnicas, sino también, y en un sentido más central, como un «enriquecimiento» general de la persona a través de la información, la reflexión crítica y la imaginación (Nussbaum, 2012, 318).

El principio «mínimo social básico» que propone se define en 10 capacidades centrales, de obligado cumplimiento para todos los Gobiernos en relación a «todos» sus ciudadanos, como condición ineludible de justicia social:

1) Vida. Disfrutar de una vida de duración normal.

2) Salud física. Mantener una buena salud.

3) Integridad física. Desplazarse libremente de un lugar a otro y estar protegido de ataques violentos.

4) Sentidos, imaginación y pensamiento. Cultivar a través de la educación los sentidos, la imaginación y el pensamiento.

5) Emociones. Poder sentir apego por las cosas y poder amar a quienes nos aman.

6) Razón práctica. Poder formarse una concepción del bien y reflexionar críticamente.

7) Afiliación. Poder vivir con y para los demás. Fomentar el respeto por nosotros mismos.

8) Otras especies. Poder vivir una relación próxima y respetuosa con los animales, las plantas y el mundo natural.

9) Juego. Poder reír, jugar y disfrutar de actividades recreativas.

10) Control sobre el propio entorno. Poder participar de forma efectiva en las decisiones políticas. Poseer propiedades.

Estas capacidades que deben ser desarrolladas en todos los ciudadanos por encima de un «umbral mínimo amplio» tienen -como se ha dicho- un carácter individual y se inspira en que "cada persona es un fin en sí misma».

Tal como hemos analizado en las propuestas pedagógicas revisadas, pareciera ser que en pleno siglo xxI hemos logrado identificar qué educar y a partir de qué estructuras fundamentales iniciar el proceso. La pregunta que se suscita ahora es: para qué y, lo más importante, cómo.

La respuesta a la primera pregunta es fácil de deducir: para generar capacidades humanas. Capacidades que hagan ser pleno al ser humano. Un ser humano en potencia que es ese niño que está en el aula. Sin embargo, a la segunda pregunta es más difícil responder, dado que en ese cómo partimos de la formación, actitud y voluntad (entendida como vocación) de los docentes, verdaderos «brazos de la educación». Desde esta premisa, esbozaremos algunas implicaciones pedagógicas de esta tríada: formación, voluntad y actitud. 


\section{Algunas IMPLICACIONES PEDAGÓGICAS DE LA «NUEVA ESCUELA»}

\subsection{El rol del docente}

Se entiende por rol docente el papel que desempeña el maestro como medio para lograr los fines educativos. Desde esta perspectiva, no se puede pretender cambiar el modelo y los objetivos de la educación (el qué enseñar), y continuar desarrollando el mismo rol (el cómo). En la escuela tradicional el papel del docente era acercar el conocimiento a los alumnos, más adelante el constructivismo modificó dicho rol, convirtiéndolo en un facilitador de experiencias en el aula para generar conocimiento. Además de asumir, como expone Davini (2008), la tarea de investigar y motivar. Robinson $(2015,149)$, lo define así: «Los jardineros saben que no son ellos los que hacen crecer las plantas, ni montan las raíces ni pegan las hojas ni tampoco pintan los pétalos; las plantas crecen solas. La labor del jardinero consiste en crear las condiciones óptimas para que eso suceda».

De esta manera, el rol es probar ante el alumnado que ese conocimiento, que fácilmente puede adquirirse en los libros, internet y otras fuentes..., es verídico, sólido, contrastable y -sobre todo- útil. Además, supone una estructura de investigación, delimita un método y exige una serie de criterios a desarrollar ya no sólo para que el alumno conozca, sino para que sepa discernir, evaluar, decidir y utilizar. Estos son los nuevos verbos que acompañan la propuesta pedagógica del siglo XXI.

\subsection{La relación profesor-alumno}

La educación es una producción de la socialización humana, por lo que la forma como un docente se relaciona con sus alumnos dice mucho no sólo de su saber, sino de su ser. Es por esta razón que el maestro establece una relación con sus alumnos y crea un clima diferente de aula, que navega entre: "ni cuidados maternales, ni abandono, la verdadera enseñanza a todos los niveles adopta a la vez el carácter inquietante del encuentro con lo desconocido y el apoyo que aporta la tranquilidad necesaria", como dice Meirieu (2011, 25). En este sentido, el docente es un desarrollador de capacidades y destrezas, donde su relación con el alumnado debe ser de máximo respeto a la diversidad y la diferencia, de atención a los ritmos de aprendizaje, de retar constantemente a sus alumnos y "su tarea reside en "crear el enigma"», o, más exactamente, en hacer saber un enigma: decir o enseñar lo suficiente [...], y callarse a tiempo para despertar el interés por el descubrimiento» (Meirieu, 2002, 100-101). Todo ello, con un profundo sentido de empatía y afectividad para comprender las diferentes realidades de sus estudiantes. 


\subsection{La atención a la diversidad}

Si hay algo concreto y físico que diferencia las aulas del siglo xxI de las del siglo pasado es la presencia de alumnos diversos y la imperiosa necesidad de entender la singularidad de estos desde un enfoque inclusivo. Es por esta razón que se habla de la urgencia de atender las necesidades educativas y sociales del alumnado para adecuar el proceso a las personas y no a la inversa. Hace algunos años, Gimeno Sacristán $(2000,68)$ definía la atención a la diversidad como «una escolaridad igual para sujetos diferentes en una escuela común».

El enfoque inclusivo es mucho más que el reconocimiento de una diversidad cultural, social e individual, supone la relevancia cultural y la significación de los aprendizajes que tienen lugar en la escuela. Para un enfoque inclusivo es importante no igualar el aprendizaje, sino comprender la procedencia social y cultural de cada alumno, así como sus características individuales:

Se trata de que tanto la persona como la sociedad asuman las características singulares de cada ser humano y se valoren positivamente dichas diferencias, ya que éstas nos enriquecen. La valoración negativa de las diferencias y los estereotipos conducen a la exclusión y la discriminación [...] En este sentido, se podría decir que la educación tiene una deuda pendiente con el respeto de las múltiples identidades y opciones personales, dada la uniformidad de la respuesta educativa que caracteriza a los sistemas educacionales (UNESCO, 2008, 19).

No se puede hablar de atención a la diversidad en un centro que parte de la premisa de crear grupos homogéneos, porque se pretenden entonces resultados homogéneos y conducen al fracaso escolar. "Ahora sabemos bien -nos dice Meirieu- que el hecho de aislar sistemáticamente a los alumnos con dificultades puede hacerlos entrar en una espiral de fracasos, pero también que tratándolos "igual" que los demás estamos incrementando un poco más las diferencias" (2002, 176). Por tanto, un centro que no atiende y cuida la diversidad desde un enfoque inclusivo es un centro escolar que no tiene calidad educativa, que no responde a los retos explícitos de la educación para el siglo XXI (Booth y Ainscow, 2015).

Un centro y un aula que parten de la base de trabajar con grupos homogéneos (falsos por principio, ya que nuestro alumnado ni es ni debería ser igual) genera contextos segregados que no responden a las individualidades y promueve una educación despersonalizada que deja al margen a todo el que no se ajusta a ese pretendido estereotipo imaginario. Supone, a su vez, dejar de considerar la educación inclusiva como «un favor que se hace a esa persona con discapacidad» y entender que dotar de apoyos y diversidad de recursos a la escuela es la forma concreta de responder a un derecho que tiene la persona (Flórez, 2016). De este modo, la educación atenderá verdaderamente a la inclusión y a la diversidad y pasará de ser un eslogan a una realidad.

Permitir una educación para la diversidad es respetar que hay diversas formas de funcionar, diversas formas de aprender, que los procesos de aprendizaje 
pueden llegar a ser tan diversos y enriquecedores como la biodiversidad de alumnado y como su diversidad funcional.

\subsection{Las metodologías de enseñanza}

En nuestro sistema educativo la brújula da vueltas sin sentido cuando hablamos de metodologías. Hay tantas y tan diversas como profesorado. La metodología de enseñanza-aprendizaje en un centro debe estar determinada por varios factores, pero -ineludiblemente- debe basarse en un estudio antropológico del perfil del alumnado al que responde y en un diagnóstico contextual para responder en justicia al significado de la educación.

Las metodologías son instrumentos que facilitan el aprendizaje, por tanto, están al servicio del mismo. No pueden ni deben ser un "corsé» en el que se "ata" al alumnado conduciéndolo al fracaso escolar.

La respuesta a la pregunta ¿cómo educar? supone el uso de metodologías activas con participación del alumnado, donde los niños son autores de su propio proceso, basado en una pedagogía centrada en la persona, donde importan sus conocimientos previos, su historia personal, su ritmo de aprendizaje y la diversidad de sus necesidades y habilidades. Estos aspectos deben ser hoy la premisa de las metodologías docentes. Los actuales retos educativos exigen una metodología basada en el reconocimiento de la plasticidad de la capacidad cerebral, que es indeterminada para crear y crecer. En la que el desarrollo cerebral va creando nuevas conexiones neuronales para que el mapa de aprendizaje se dé a partir de un proceso de significados, basado en retos y enigmas (Jensen, 2005, 2008).

\subsection{El clima de aula}

Cada vez existen más evidencias de los efectos de un clima positivo de aula en los procesos de aprendizaje. Es así como las teorías sobre neuroaprendizaje indican su importancia sobre la actitud de los alumnos al aprender. Los resultados de diversos estudios (Pritchard, 2014) identifican varios factores asociados de forma positiva al contexto del aula: las características de personalidad docente; su estilo; el tipo de relaciones y roles; así como la disposición y el ambiente físico de la propia clase.

Así, por ejemplo, aspectos tan sencillos como la disposición de espacios más informales, grupos de trabajo en círculo... son percibidos por los alumnos como contextos distendidos que favorecen las relaciones interpersonales de forma más relajada, más plausibles que los espacios de líneas rectas o formas cuadráticas, interpretados como jerárquicos y con distancia cognitiva entre docentes y alumnos. Además, se interpretan según la cercanía del "lugar físico» como de mejor o mayor accesibilidad al docente. 
Otro aspecto fundamental para crear aulas positivas, según las nuevas propuestas del neuroaprendizaje, es la creación de relaciones estables y seguras, donde todos conocen las reglas de participación y disfrutan de un ambiente relajado en igualdad de condiciones para el aprendizaje. Aulas en las que un docente dinamizador de retos está fuera del estrés permanente de sentirse a "prueba" o evaluado. En otras palabras, el clima de aula más positivo es aquel que evidencia que cada alumno aporta algo a la construcción global del aprendizaje y donde cualquier aporte es valorado como forma de añadir o cuestionar espontáneamente el contenido trabajado (Mora, 2013). En esa línea, la creación de espacios emocionalmente saludables, en los que la experiencia en el aula se basa en un aprendizaje emocional, cargado de significados para la vida y donde se requiere la gestión de tiempos, intereses, emociones..., se establece como necesario en la escuela del siglo XXI.

\section{CONCLUSiOnes}

Para concluir, presentamos una serie de retos a los que debe responder la educación:

1. Reto de la calidad educativa. Reconocer el derecho a una educación de calidad universal, basado en un modelo inclusivo, que atienda a las singularidades del alumnado; y que, partiendo de la plasticidad cerebral para el aprendizaje, ofrezca un modelo educativo interactivo, emocional y dinámico, en el que se reorienten los aprendizajes hacia el desarrollo de capacidades que permitan a las personas enfrentarse a la vida.

2. Reto de una educación bumanizadora. Se trata de bumanizar la escuela en un doble sentido. Por un lado, implementando aquellos aprendizajes que convierten al individuo en ser humano, desarrollando todas aquellas actitudes y aptitudes humanas que posibilitan el respeto a la vida, la ecología y la diversidad y, por otro lado, dotándola de sentimiento. Una escuela que siente, que se preocupa y ocupa de todos sus alumnos, que sitúa las emociones en el centro del proceso educativo, que atiende a las diferencias -de capacidades, de ritmos, de estilos de aprendizaje- y de historias personales.

3. Reto de una escuela fértil. En la que los contenidos están cargados de significados. Una educación marcada por el desarrollo de las potencialidades, en la que los currículos están relacionados con la vida y la capacidad que demanda el contexto social, cultural y económico. Un modelo basado en el progreso de las culturas y en un enfoque integral sostenible.

4. Reto de una metodología becha con conciencia de aprendizaje. En la que la forma o el cómo están determinados por la antropología social y cultural del contexto de los centros escolares, que no sólo cambia el sentido de lo que enseña, sino también la forma en la que lo enseña. 
5. Reto de una educación dinámica. Que crece, cambia y se dinamiza. Que reconoce que la investigación en psicología, pedagogía y la vida tienen mucho que decir en qué y cómo es la educación. Una dinámica educativa que se basa en los estudios de la neurociencia, que estudian, investigan, proponen y adaptan las nuevas formas de pensar del alumnado a los cambios de la historia y a los retos de las ciencias de la información y la tecnología.

6. Reto para crecer juntos en el conocimiento. En el que el clima del aula sitúa al alumno en el centro del proceso, que utiliza metodologías activas -fundamentadas en la experiencia vivida y reflexionada- y cooperativas, buscando despertar los talentos de cada uno y no enfrentando a los alumnos frente a los resultados académicos. Que trabaja por proyectos, de manera globalizada, atendiendo a la diversidad y gestionando de manera positiva los conflictos en el aula.

7. Reto de un nuevo rol docente. Que reconoce que en el proceso educativo se cambia cada día... y que se crece como persona en una interrelación con otros. Donde su rol no está determinado por una zona de confort, sino que cambia y acepta la zona de riesgo "de aprender", en donde se es verdaderamente un auctoritas que mantiene la admiración de los alumnos, la sorpresa, el asombro por el saber y el profundo respeto por su profesión como modelo.

8. Reto de una evaluación constructiva. En la que no se acepta el fracaso escolar como posibilidad, no se abandona al que lleva otro ritmo, no se permite el abandono y el absentismo. En el que la escuela no se contenta con saber que sabe, sino que cumple la función de hacer que el saber llegue a todos.

En definitiva, como nos dice Santos Guerra (2012, 17), "para aprender de forma eficaz hace falta tener deseos de hacerlo y tener los ojos abiertos para ver, la mente despierta para analizar, el corazón dispuesto para asimilar lo aprendido y los brazos prestos para aplicarlo».

\section{REFERENCIAS BIBLIOGRÁFICAS}

Armstrong, T. (2012) El poder de la neurodiversidad. Barcelona, Paidós.

Bernal, A. (2014) La función de la educación para la creación de las capacidades centrales. Edetania. Estudios y Propuestas Socieducativas, 46, 123-140.

Bokova, I. (2015) Replantear la educación. ¿Hacia un bien común mundial? París, unesco. Booth, T. y Ainscow, M. (2015) Guía para la educación inclusiva. Desarrollando el aprendizaje y la participación en los centros escolares. Madrid, FuHEM-OEI.

Davini, M. (2008) Métodos de enseñanza: didáctica general para maestros y profesores. Buenos Aires, Santillana.

Delors, J. (1996) La educación encierra un tesoro. París, unesco. 
Escámez, J. (2004) La educación para la promoción de los derechos humanos de la 3 a $^{\text {a }}$ generación. Encounters on Education, vol. 5, 81-100.

FAure, E. (1973) Aprender a ser. La educación del futuro. París, UNESCO.

FERnÁNDEZ COTO, R. (2012) Cerebrando el aprendizaje: Recursos teórico-prácticos para conocer y potenciar el "órgano del aprendizaje». Buenos Aires, Bonum.

FlóREZ, J. (2016) Neurodiversidad e inteligencias múltiples. Revista Española de Investigación e Información sobre el Sindrome de Down, 129, 59-64.

Gardner, H. (2015a) Inteligencias múltiples. La teoría en la práctica. Barcelona, Paidós.

Gardner, H. (2015b) Las cinco mentes del futuro. Barcelona, Paidós.

GIMENO SACRISTÁN, J. (2000) La educación obligatoria: su sentido educativo y social. Madrid, Morata.

Jensen, E. (2005) Teaching with the brain in mind. USA, Association for Supervision and Curriculum Development.

Jensen, E. (2008) Exploring exceptional brains, en The Jossey-Bass Reader on the Brain and Learning. San Francisco, Wiley, 385-404.

Marina, J. A. (2015) Despertad al diplodocus. Una conspiración educativa para transformar la escuela... y todo lo demás. Barcelona, Ariel.

Meirieu, P. (2002) Aprender, sí pero ¿cómo? Barcelona, Octaedro.

MeIRIEu, P. (2003) Frankenstein educador. Barcelona, Laertes.

Meirieu, P. (2011) Carta a un joven profesor. Por qué enseñar hoy. Barcelona, Graó.

Mora, F. (2013) Neuroeducación. Madrid, Alianza.

Morin, E. (2000) Los siete saberes necesarios para la educación del futuro. París, UNESCO.

NaCiones Unidas (1948) Declaración Universal de Derechos Humanos. París, Aegitas.

Nussbaum, M. (2012) Las fronteras de la justicia. Consideraciones sobre la exclusión. Barcelona, Paidós.

Nussbaum, M. (2013) Crear capacidades. Propuesta para el desarrollo bumano. Barcelona, Paidós.

PerRenoud, P. (2012) Cuando la escuela pretende preparar para la vida. ¿Desarrollar competencias o enseñar otros saberes? Barcelona, Graó.

PNUD (2004) La libertad cultural en el mundo diverso hoy. Madrid, PNUD.

PNUD (2005) La cooperación internacional ante una encrucijada: Ayuda al desarrollo, comercio y seguridad en un mundo desigual. Madrid, PNUD.

PNUD (2009) Superando barreras: Movilidad y desarrollo humanos. Madrid, PNUD.

PNUD (2010) La verdadera riqueza de las naciones: caminos al desarrollo bumano. Madrid, PNUD.

PRITCHARD, A (2014) Ways of learning: Learning theories and learning styles in the classroom. U. K., Routledge.

Robinson, K. (2015) Escuelas creativas. La revolución que está trasformando la educación. Barcelona, Grijalbo.

Rodríguez, L. (2006) Ética de la vida buena. Bilbao, Desclée.

SAntos Guerra, M. A. (2012) La escuela que aprende. Madrid, Morata.

SANZ, R. y SERrano, A. (2016) El desarrollo de capacidades en la educación. Una cuestión de Justicia Social. Sinéctica. Revista Electrónica de Educación, 46, 1-16.

uNESCO (2008) Educación y diversidad cultural. Lecciones desde la práctica innovadora de América Latina. Santiago de Chile, Pehúen. 